\title{
ON A CAMERON-MARTIN TYPE QUASI-INVARIANCE THEOREM AND APPLICATIONS TO SUBORDINATE BROWNIAN MOTION
}

\author{
CHANG-SONG DENG AND RENÉ L. SCHILLING
}

\begin{abstract}
We present a Cameron-Martin type quasi-invariance theorem for subordinate Brownian motion. As applications, we establish an integration by parts formula and construct a gradient operator on the path space of subordinate Brownian motion, and we obtain some canonical Dirichlet forms. These findings extend the corresponding classical results for Brownian motion.
\end{abstract}

\section{INTRODUCTION}

Recently the stability of properties of Markov processes and their semigroups under subordination in the sense of Bochner has attracted great interest. In [6], Wang's dimension free Harnack inequality was established for a class of subordinate semigroups. Nash and Poincaré inequalities are preserved under subordination, cf. [13, 5]. In our recent paper [2], we show that shift Harnack inequalities (in the sense of [16]) remain valid under subordination in the sense of Bochner. It is a natural question whether further probabilistic properties, e.g. quasi-invariance, are preserved by subordination.

The Cameron-Martin theorem, which was discovered by R.H. Cameron and W.T. Martin [1] (see e.g. [3, 7, 8] and the references therein for further developments), plays a fundamental role in the analysis on the path space of diffusion processes. It states that the Wiener measure (i.e. the distribution of Brownian motion) is quasi-invariant under a Cameron-Martin shift. In this paper, we shall derive an analogous result for subordinate Brownian motion.

Let us recall some basic notations. Throughout this paper, we set

$$
[0, A]:= \begin{cases}\{x: 0 \leq x \leq A\}, & \text { if } A<\infty \\ \{x: 0 \leq x<\infty\}, & \text { if } A=\infty\end{cases}
$$

and make the convention

$$
\int_{u}^{v}=\int_{(u, v)} \text { for all } 0 \leq u<v \leq \infty .
$$

By $S=\left(S_{t}\right)_{t \in[0, T]}$, where $0<T \leq \infty$, we denote a non-trivial subordinator, i.e. an increasing Lévy process with $S_{0}=0$ and Laplace transform

$$
\mathbb{E} \mathrm{e}^{-u S_{t}}=\mathrm{e}^{-t \phi(u)}, \quad u>0, t \in[0, T] .
$$

Date: September 8, 2018.

2010 Mathematics Subject Classification. 60J75, 60G17.

Key words and phrases. Cameron-Martin theorem, quasi-invariance, integration by parts formula, subordinate Brownian motion.

The first-named author gratefully acknowledges support through the Alexander-von-Humboldt foundation, the National Natural Science Foundation of China (11401442) and the International Postdoctoral Exchange Fellowship Program (2013). 
The characteristic (Laplace) exponent $\phi$ is a Bernstein function with $\phi(0+)=0$; all such exponents are completely characterized by the following Lévy-Khintchine formula

$$
\phi(u)=b u+\int_{0}^{\infty}\left(1-\mathrm{e}^{-u x}\right) \nu(\mathrm{d} x), \quad u>0,
$$

where $b \geq 0$ is the drift parameter and $\nu$ is a Lévy measure, i.e. a measure on $(0, \infty)$ satisfying $\int_{0}^{\infty}(x \wedge 1) \nu(\mathrm{d} x)<\infty$; we use [12] as our standard reference for Bernstein functions and subordination. If $T=\infty$ then

$$
S_{\infty}:=\lim _{t \uparrow \infty} S_{t}=\infty \quad \text { a.s. }
$$

Let

$$
M=\operatorname{ess} \sup S_{T}=\sup \left\{r>0: \mathbb{P}\left(S_{T}<r\right)<1\right\} .
$$

Remark 1.1. $M$ can attain the following values:

(i) If $T=\infty$, then $S_{\infty}=\infty$ a.s. and $M=\infty$.

(ii) If $T<\infty$ and $S_{t}$ is deterministic, i.e. $S_{t}=c t$ for some constant $c>0$, then $M=c T<\infty$.

(iii) If $T<\infty$ and $S_{t}$ is non-deterministic, then $M=\infty$.

Indeed: Since $\nu \neq 0$, there exists some finite interval $[u, v] \subset(0, \infty)$ such that $\eta:=\nu([u, v]) \in(0, \infty)$. The jump times of jumps with size in the interval $[u, v]$ define a Poisson process $\left(N_{t}\right)_{t \in[0, T]}$ with intensity $\eta$. Since $S_{T} \geq u N_{T}$, we conclude that $\operatorname{ess} \sup S_{T}=\infty$.

Let $\left(W_{t}\right)_{t \in[0, M]}$ be a standard $d$-dimensional Brownian motion starting from zero. The Wiener measure $\mu$, i.e. the distribution of $\left(W_{t}\right)_{t \in[0, M]}$, is a probability measure on the path space

$$
\mathbb{W}_{M}=\left\{w:[0, M] \rightarrow \mathbb{R}^{d}: w \text { is continuous and } w(0)=0\right\},
$$

which is endowed with the topology of locally uniform convergence. We write

$$
\mathbb{H}_{M}:=\left\{h \in \mathbb{W}_{M}: h \text { is absolutely continuous and } h^{\prime} \in L^{2}\left([0, M] ; \mathbb{R}^{d}\right)\right\}
$$

for the Cameron-Martin space; $\mathbb{H}_{M}$ is a Hilbert space with the inner product

$$
\langle g, h\rangle_{\mathbb{H}_{M}}:=\int_{0}^{M}\left\langle g^{\prime}(t), h^{\prime}(t)\right\rangle_{\mathbb{R}^{d}} \mathrm{~d} t, \quad g, h \in \mathbb{H}_{M} .
$$

Let $h \in \mathbb{W}_{M}$ and denote by $\mu_{h}$ the distribution of $\left(W_{t}+h(t)\right)_{t \in[0, M]}$. Then the CameronMartin theorem says that $\mu$ and $\mu_{h}$ are equivalent (i.e. mutually absolutely continuous) if, and only if, $h \in \mathbb{H}_{M}$; in this case

$$
\frac{\mathrm{d} \mu_{h}}{\mathrm{~d} \mu}=\exp \left[\int_{0}^{M} h^{\prime}(t) \mathrm{d} W_{t}-\frac{1}{2} \int_{0}^{M}\left|h^{\prime}(t)\right|^{2} \mathrm{~d} t\right] .
$$

Throughout this paper, we assume that $\left(S_{t}\right)_{t \in[0, T]}$ is independent of the standard Brownian motion $\left(W_{t}\right)_{t \in[0, M]}$ on $\mathbb{R}^{d}$. The process $W_{S}=\left(W_{S_{t}}\right)_{t \in[0, T]}$ is called a subordinate Brownian motion; it is a rotationally invariant Lévy process with characteristic (Fourier) exponent (symbol) $\phi\left(|\xi|^{2} / 2\right)=-\log \mathbb{E e}^{i\left\langle\xi, W_{S_{1}}\right\rangle_{\mathbb{R}^{d}}}$.

Inspired by the quasi-invariance property of the Wiener measure under CameronMartin shifts, we are interested in the following problem: Let $\xi=\left(\xi_{t}\right)_{t \in[0, T]}$ be a further (random) function and consider the perturbation $W_{S}+\xi=\left(W_{S_{t}}+\xi_{t}\right)_{t \in[0, T]}$ of the subordinate Brownian motion $W_{S}=\left(W_{S_{t}}\right)_{t \in[0, T]}$. For which (random) perturbations are the distributions of $W_{S}$ and $W_{S}+\xi$ equivalent? Related results for compound Poisson processes can be found in [17] and [15]. In this note, we assume that $\xi_{t}=h\left(S_{t}\right)$ where $h \in \mathbb{W}_{M}$ and $t \in[0, T]$. Let $\mu^{S}$ and $\mu_{h}^{S}$ be the distributions of $\left(W_{S_{t}}\right)_{t \in[0, T]}$ and $\left(W_{S_{t}}+h\left(S_{t}\right)\right)_{t \in[0, T]}$, 
respectively. We aim to find sufficient and necessary conditions so that $\mu_{h}^{S}$ is equivalent to $\mu^{S}$.

Our problem can also be seen as a stability property for Bochner's subordination: Under which circumstances is the quasi-invariance on Wiener space inherited by the subordinate (i.e. time-changed) process?. For a deterministic subordinator $S$, this is just the classical Cameron-Martin theorem. For a general subordinator we need to assume some additional conditions in order to ensure that quasi-invariance is preserved.

This paper is organized in the following way: First, we establish the Cameron-Martin type theorem for subordinate Brownian motion in Section 2 . If $h$ is absolutely continuous, $h(0)=0$ and $\int_{0}^{S_{T}}\left|h^{\prime}(t)\right|^{2} \mathrm{~d} t<\infty$ almost surely, then $\mu_{h}^{S}$ is equivalent to $\mu^{S}$. The crucial point in our proof is that a functional on the path space of a subordinate Brownian motion is also a functional on the classical Wiener space. Once we have established the quasiinvariance property, we can derive in Section 3 an integration by parts formula. A natural gradient operator $D^{(\kappa)}$ is defined for $\kappa \in \mathbb{R}$ on the family of cylinder functions $\mathscr{F} C_{b}^{\infty}$ through the Riesz representation theorem. Furthermore, we characterize the gradient operator $D^{(\kappa)}$; as applications we construct some natural Dirichlet forms on the path space of subordinate Brownian motion. In the final section, we present the detailed proof of a result used in Section 3, which describes the subordinator index of $S$ at the origin in terms of the underlying Lévy measure (cf. [11] for the corresponding result for general Feller processes).

\section{Cameron-Martin type theorem}

In this section, we extend the classical Cameron-Martin theorem to subordinate Brownian motion. Let $h \in \mathbb{W}_{M}$ and write $\mu^{S}$ and $\mu_{h}^{S}$ for the distributions of $W_{S}=\left(W_{S_{t}}\right)_{t \in[0, T]}$ and $W+h(S)=\left(W_{S_{t}}+h\left(S_{t}\right)\right)_{t \in[0, T]}$, respectively. Denote by $A C\left([0, M] ; \mathbb{R}^{d}\right)$ the family of all absolutely continuous functions from $[0, M]$ to $\mathbb{R}^{d}$. The following Cameron-Martin type space will be important $(\kappa \in \mathbb{R})$ :

$$
\mathbb{H}_{M}^{(\kappa)}:=\left\{h \in \mathbb{W}_{M} \cap A C\left([0, M] ; \mathbb{R}^{d}\right): \int_{0}^{M}\left|h^{\prime}(t)\right|^{2}\left[\mathbb{P}\left(S_{T} \geq t\right)\right]^{\kappa} \mathrm{d} t<\infty\right\},
$$

which becomes a Hilbert space with the inner product

$$
\langle g, h\rangle_{\mathbb{H}_{M}^{(\kappa)}}:=\int_{0}^{M}\left\langle g^{\prime}(t), h^{\prime}(t)\right\rangle_{\mathbb{R}^{d}}\left[\mathbb{P}\left(S_{T} \geq t\right)\right]^{\kappa} \mathrm{d} t, \quad g, h \in \mathbb{H}_{M}^{(\kappa)} .
$$

As usual, we set $0^{0}:=1$. It is clear that $\mathbb{H}_{M}^{(0)}=\mathbb{H}_{M}$ and $\mathbb{H}_{M}^{(\kappa)}$ is non-decreasing in $\kappa$, i.e. $\kappa_{1} \leq \kappa_{2}$ implies $\mathbb{H}_{M}^{\left(\kappa_{1}\right)} \subset \mathbb{H}_{M}^{\left(\kappa_{2}\right)}$. A direct calculation shows that

$$
\int_{0}^{M}\left\langle g^{\prime}(t), h^{\prime}(t)\right\rangle_{\mathbb{R}^{d}}\left[\mathbb{P}\left(S_{T} \geq t\right)\right]^{\kappa} \mathrm{d} t=\mathbb{E}\left[\int_{0}^{S_{T}}\left\langle g^{\prime}(t), h^{\prime}(t)\right\rangle_{\mathbb{R}^{d}}\left[\mathbb{P}\left(S_{T} \geq t\right)\right]^{\kappa-1} \mathrm{~d} t\right]
$$

for $g, h \in \mathbb{H}_{M}^{(\kappa)}$. Therefore,

$$
h \in \mathbb{H}_{M}^{(1)} \quad \Longrightarrow \quad \int_{0}^{S_{T}}\left|h^{\prime}(t)\right|^{2} \mathrm{~d} t<\infty \quad \text { almost surely. }
$$

Remark 2.1. a) If either $T=\infty$ or $T<\infty$ and $M<\infty$, then $\mathbb{H}_{M}^{(\kappa)}$ does not depend on $\kappa$ and $\mathbb{H}_{M}^{(\kappa)}=\mathbb{H}_{M}$ for all $\kappa \in \mathbb{R}$.

b) If $T<\infty$ and $M=\infty$, then $\mathbb{H}_{M}^{(\kappa)}$ is strictly increasing in $\kappa \in \mathbb{R}$, see Example 2.2 below. 
Example 2.2. Let $T<\infty, M=\infty$ and $\kappa_{1}<\kappa_{2}$. We will show that $\mathbb{H}_{M}^{\left(\kappa_{2}\right)} \backslash \mathbb{H}_{M}^{\left(\kappa_{1}\right)} \neq \emptyset$. Since $\mathbb{P}\left(S_{T} \geq t\right) \downarrow 0$ as $t \uparrow \infty$, we have

$$
[0, \infty)=\bigcup_{m=1}^{\infty} B_{m}
$$

where

$$
B_{m}:=\left\{t \in[0, \infty): \frac{1}{(m+1)^{3}}<\left[\mathbb{P}\left(S_{T} \geq t\right)\right]^{\kappa_{2}-\kappa_{1}} \leq \frac{1}{m^{3}}\right\}, \quad m \in \mathbb{N},
$$

are mutually disjoint bounded Borel measurable sets. Put

$$
\Phi(\mathrm{d} t):=\left[\mathbb{P}\left(S_{T} \geq t\right)\right]^{\kappa_{1}} \mathrm{~d} t .
$$

It is easy to see that $\Phi\left(B_{m}\right)<\infty$ for each $m \in \mathbb{N}$ and

$$
\#\left\{m \in \mathbb{N}: \Phi\left(B_{m}\right)>0\right\}=\infty .
$$

Pick $h \in \mathbb{W}_{\infty} \cap A C\left([0, \infty) ; \mathbb{R}^{d}\right)$ such that

$$
\left|h^{\prime}(t)\right|=\sum_{m: \Phi\left(B_{m}\right)>0} \mathbb{1}_{B_{m}}(t) \sqrt{\frac{m}{\Phi\left(B_{m}\right)}}, \quad t \geq 0 .
$$

Then

$$
\begin{aligned}
\int_{0}^{\infty}\left|h^{\prime}(t)\right|^{2}\left[\mathbb{P}\left(S_{T} \geq t\right)\right]^{\kappa_{2}} \mathrm{~d} t & =\sum_{m=1}^{\infty} \int_{B_{m}}\left|h^{\prime}(t)\right|^{2}\left[\mathbb{P}\left(S_{T} \geq t\right)\right]^{\kappa_{2}-\kappa_{1}} \Phi(\mathrm{d} t) \\
& \leq \sum_{m: \Phi\left(B_{m}\right)>0} \int_{B_{m}} \frac{m}{\Phi\left(B_{m}\right)} \cdot \frac{1}{m^{3}} \Phi(\mathrm{d} t) \\
& =\sum_{m: \Phi\left(B_{m}\right)>0} \frac{1}{m^{2}} \\
& \leq \sum_{m=1}^{\infty} \frac{1}{m^{2}}<\infty
\end{aligned}
$$

whereas

$$
\begin{aligned}
\int_{0}^{\infty}\left|h^{\prime}(t)\right|^{2}\left[\mathbb{P}\left(S_{T} \geq t\right)\right]^{\kappa_{1}} \mathrm{~d} t & =\sum_{m: \Phi\left(B_{m}\right)>0} \int_{B_{m}}\left|h^{\prime}(t)\right|^{2} \Phi(\mathrm{d} t) \\
& =\sum_{m: \Phi\left(B_{m}\right)>0} \int_{B_{m}} \frac{m}{\Phi\left(B_{m}\right)} \Phi(\mathrm{d} t) \\
& =\sum_{m: \Phi\left(B_{m}\right)>0} m=\infty .
\end{aligned}
$$

This means that $h \in \mathbb{H}_{M}^{\left(\kappa_{2}\right)} \backslash \mathbb{H}_{M}^{\left(\kappa_{1}\right)}$.

Theorem 2.3. If $h \in \mathbb{W}_{M} \cap A C\left([0, M] ; \mathbb{R}^{d}\right)$ and $\int_{0}^{S_{T}}\left|h^{\prime}(t)\right|^{2} \mathrm{~d} t<\infty$ almost surely (e.g. if $h \in \mathbb{H}_{M}^{(1)}$ ), then $\mu_{h}^{S}$ and $\mu^{S}$ are equivalent; moreover,

$$
\frac{\mathrm{d} \mu_{h}^{S}}{\mathrm{~d} \mu^{S}}=\exp \left[\int_{0}^{S_{T}} h^{\prime}(t) \mathrm{d} W_{t}-\frac{1}{2} \int_{0}^{S_{T}}\left|h^{\prime}(t)\right|^{2} \mathrm{~d} t\right] .
$$

Remark 2.4. If $S_{t} \equiv t$ for all $t \in[0, T]$, then $M=T \in(0, \infty]$ and Theorem 2.3 reduces to the classical Cameron-Martin theorem.

Theorem 2.5. Let $h \in \mathbb{W}_{M}$. If $\mu_{h}^{S}$ and $\mu^{S}$ are equivalent, then $h \in \mathbb{H}_{M}$. 
Since $\mathbb{H}_{M}=\mathbb{H}_{M}^{(0)} \subset \mathbb{H}_{M}^{(1)}$, the following result is a direct consequence of Theorems 2.3 and 2.5 .

Corollary 2.6. Assume that $h \in \mathbb{W}_{M}$. Then $\mu_{h}^{S} \sim \mu^{S}$ if, and only if, $h \in \mathbb{H}_{M}$.

For the proof of Theorem 2.3 we need a few preparations. Note that $\left(W_{t}\right)_{t \in[0, M]}$ can be regarded as a process on the classical Wiener space $\left(\mathbb{W}_{M}, \mathscr{B}\left(\mathbb{W}_{M}\right), \mu\right)$ :

$$
W_{t}(w):=w(t), \quad t \in[0, M], w \in \mathbb{W}_{M} .
$$

Let $\lambda$ be the distribution of $\left(S_{t}\right)_{t \in[0, T]}$, which is a probability measure on the path space

$$
\mathbb{S}_{T}:=\left\{\ell:[0, T] \rightarrow[0, \infty): \ell_{0}=0 \text {, increasing and càdlàg }\right\},
$$

which we equip with the Skorokhod topology. Thus, the subordinator $\left(S_{t}\right)_{t \in[0, T]}$ can be realized as a canonical process on $\left(\mathbb{S}_{T}, \mathscr{B}\left(\mathbb{S}_{T}\right), \lambda\right)$ :

$$
S_{t}(\ell):=\ell_{t}, \quad t \in[0, T], \ell \in \mathbb{S}_{T} .
$$

Since $S$ and $W$ are independent, $\left(W_{S_{t}}\right)_{t \in[0, T]}$ is the canonical process on the product space $\left(\mathbb{W}_{M} \times \mathbb{S}_{T}, \mathscr{B}\left(\mathbb{W}_{M}\right) \otimes \mathscr{B}\left(\mathbb{S}_{T}\right), \mu \times \lambda\right)$ :

$$
W_{S_{t}}(w, \ell):=W_{S_{t}(\ell)}(w)=w\left(\ell_{t}\right), \quad t \in[0, T], w \in \mathbb{W}_{M}, \ell \in \mathbb{S}_{T} .
$$

Moreover, $\mu^{S}$ is is a probability measure on the path space

$$
\Omega:=\left\{w \circ \ell: w \in \mathbb{W}_{M}, \ell \in \mathbb{S}_{T}\right\}
$$

equipped with the Skorokhod topology. If $T=\infty$, we set

$$
\ell_{\infty}:=\lim _{t \uparrow \infty} \ell_{t}, \quad \ell \in \mathbb{S}_{\infty} .
$$

Proof of Theorem 2.3. a) We are going to show that

$$
\begin{aligned}
& \iint_{\mathbb{W}_{M} \times \mathbb{S}_{T}} F(w \circ \ell+h \circ \ell) \mu(\mathrm{d} w) \lambda(\mathrm{d} \ell) \\
& \quad=\iint_{\mathbb{W}_{M} \times \mathbb{S}_{T}} F(w \circ \ell) \exp \left[\int_{0}^{\ell_{T}} h^{\prime}(t) \mathrm{d} W_{t}(w)-\frac{1}{2} \int_{0}^{\ell_{T}}\left|h^{\prime}(t)\right|^{2} \mathrm{~d} t\right] \mu(\mathrm{d} w) \lambda(\mathrm{d} \ell)
\end{aligned}
$$

holds for every bounded measurable function $F$ on $\Omega$. Using a standard monotone class argument, it is enough to check this equality for cylinder functions of the form

$$
F(w \circ \ell)=f\left(w\left(\ell_{t_{1}}\right), \ldots, w\left(\ell_{t_{n}}\right)\right), \quad w \circ \ell \in \Omega,
$$

where $n \in \mathbb{N}, t_{1}, \ldots, t_{n} \in[0, T], 0<t_{1}<\cdots<t_{n}$ and $f \in C_{b}\left(\mathbb{R}^{d \cdot n}\right)$. Therefore, it remains to show that

$$
\begin{aligned}
& \iint_{\mathbb{W}_{M} \times \mathbb{S}_{T}} f\left(w\left(\ell_{t_{1}}\right)+\right.\left.h\left(\ell_{t_{1}}\right), \ldots, w\left(\ell_{t_{n}}\right)+h\left(\ell_{t_{n}}\right)\right) \mu(\mathrm{d} w) \lambda(\mathrm{d} \ell) \\
&=\iint_{\mathbb{W}_{M} \times \mathbb{S}_{T}} f\left(w\left(\ell_{t_{1}}\right), \ldots, w\left(\ell_{t_{n}}\right)\right) \\
& \times \exp \left[\int_{0}^{\ell_{T}} h^{\prime}(t) \mathrm{d} W_{t}(w)-\frac{1}{2} \int_{0}^{\ell_{T}}\left|h^{\prime}(t)\right|^{2} \mathrm{~d} t\right] \mu(\mathrm{d} w) \lambda(\mathrm{d} \ell) .
\end{aligned}
$$

b) Fix $\ell \in \mathbb{S}_{T}$ such that $\ell_{T} \leq M$ and

$$
\int_{0}^{\ell_{T}}\left|h^{\prime}(t)\right|^{2} \mathrm{~d} t<\infty
$$


Then the classical Cameron-Martin theorem, applied to the bounded measurable function on $\mathbb{W}_{\ell_{T}}$ :

$$
w \mapsto f\left(w\left(\ell_{t_{1}}\right), \ldots, w\left(\ell_{t_{n}}\right)\right)
$$

yields

$$
\begin{array}{rl}
\int_{\mathbb{W}_{M}} & f\left(w\left(\ell_{t_{1}}\right)+h\left(\ell_{t_{1}}\right), \ldots, w\left(\ell_{t_{n}}\right)+h\left(\ell_{t_{n}}\right)\right) \mu(\mathrm{d} w) \\
& =\int_{\mathbb{W}_{\ell_{T}}} f\left(w\left(\ell_{t_{1}}\right)+h\left(\ell_{t_{1}}\right), \ldots, w\left(\ell_{t_{n}}\right)+h\left(\ell_{t_{n}}\right)\right) \mu(\mathrm{d} w) \\
& =\int_{\mathbb{W}_{\ell_{T}}} f\left(w\left(\ell_{t_{1}}\right), \ldots, w\left(\ell_{t_{n}}\right)\right) \exp \left[\int_{0}^{\ell_{T}} h^{\prime}(t) \mathrm{d} W_{t}(w)-\frac{1}{2} \int_{0}^{\ell_{T}}\left|h^{\prime}(t)\right|^{2} \mathrm{~d} t\right] \mu(\mathrm{d} w) \\
& =\int_{\mathbb{W}_{M}} f\left(w\left(\ell_{t_{1}}\right), \ldots, w\left(\ell_{t_{n}}\right)\right) \exp \left[\int_{0}^{\ell_{T}} h^{\prime}(t) \mathrm{d} W_{t}(w)-\frac{1}{2} \int_{0}^{\ell_{T}}\left|h^{\prime}(t)\right|^{2} \mathrm{~d} t\right] \mu(\mathrm{d} w) .
\end{array}
$$

Since our assumption implies that $\ell_{T} \leq M$ and (2.2) hold for $\lambda$-almost all $\ell \in \mathbb{S}_{T}$, we can integrate both sides of the equality with respect to $\lambda(\mathrm{d} \ell)$ to obtain (2.1). This completes the proof.

Proof of Theorem [2.5. Suppose that $h \notin \mathbb{H}_{M}$. Then it is a classical result, see e.g. [8, Proof of Theorem 8.1.5, p. 233-234], that $\mu_{h}$ and $\mu$ are mutually singular. Note that the proof in [8] uses the time interval $[0,1]$. It is not hard to see that the method used in [8] also applies to $[0, T]$ for $0<T \leq \infty$. Thus, there exists a measurable subset $A \subset \mathbb{W}_{M}$ such that

$$
\mu(A)=1, \quad \mu_{h}(A)=0 .
$$

Let

$$
\tilde{A}:=\left\{w \circ \ell \in \Omega: w \in A, \ell \in \mathbb{S}_{T}\right\}
$$

Then we have

$$
\mu^{S}(\tilde{A})=\mu(A)=1, \quad \mu_{h}^{S}(\tilde{A})=\mu_{h}(A)=0 .
$$

This, however, contradicts our assumption that $\mu_{h}^{S}$ and $\mu^{S}$ are equivalent.

\section{INTEGRATION BY PARTS FORMULA AND GRADIENT OPERATOR}

Let $h \in \mathbb{W}_{M}$. The directional derivative of a function $F$ on $\Omega$ in direction $h$ is defined as

$$
D_{h} F(w \circ \ell):=\lim _{\epsilon \rightarrow 0} \frac{F(w \circ \ell+\epsilon h \circ \ell)-F(w \circ \ell)}{\epsilon}, \quad w \circ \ell \in \Omega,
$$

whenever the limit exists. An important class of functions on $\Omega$ for which the above definition of $D_{h} F$ makes sense are the smooth cylinder functions, denoted by $\mathscr{F} C_{b}^{\infty}$, i.e. the set of all functions having the form

$$
F(w \circ \ell)=f\left(w\left(\ell_{t_{1}}\right), \ldots, w\left(\ell_{t_{n}}\right)\right), \quad w \circ \ell \in \Omega,
$$

where $n \in \mathbb{N}, f \in C_{b}^{\infty}\left(\mathbb{R}^{d \cdot n}\right)$ and $t_{1}, \ldots, t_{n} \in[0, T]$ with $t_{1}<\cdots<t_{n}$. If $F \in \mathscr{F} C_{b}^{\infty}$ is given by (3.1), then it is clear that $D_{h} F$ exists everywhere and

$$
D_{h} F(w \circ \ell)=\sum_{i=1}^{n}\left\langle\nabla_{i} f\left(w\left(\ell_{t_{1}}\right), \ldots, w\left(\ell_{t_{n}}\right)\right), h\left(\ell_{t_{i}}\right)\right\rangle_{\mathbb{R}^{d}}, \quad w \circ \ell \in \Omega,
$$

where $\nabla_{i} f$ is the gradient of $f$ w.r.t. the $i$ th variable.

First, we consider the integration by parts formula. 
Theorem 3.1. If $h \in \mathbb{W}_{M} \cap A C\left([0, M] ; \mathbb{R}^{d}\right)$ and $\int_{0}^{S_{T}}\left|h^{\prime}(t)\right|^{2} \mathrm{~d} t<\infty$ almost surely ( e.g. if $\left.h \in \mathbb{H}_{M}^{(1)}\right)$, then for any $F, G \in \mathscr{F} C_{b}^{\infty}$,

$$
\mathbb{E}\left[G D_{h} F\right]=\mathbb{E}\left[F D_{h}^{*} G\right]
$$

where

$$
D_{h}^{*} G:=-D_{h} G+G \int_{0}^{S_{T}} h^{\prime}(t) \mathrm{d} W_{t} .
$$

Proof. Using Theorem 2.3, it follows that for all $\epsilon \in \mathbb{R}$

$$
\begin{aligned}
& \iint_{\mathbb{W}_{M} \times \mathbb{S}_{T}} F(w \circ \ell+\epsilon h \circ \ell) G(w \circ \ell) \mu(\mathrm{d} w) \lambda(\mathrm{d} \ell) \\
& =\iint_{\mathbb{W}_{M} \times \mathbb{S}_{T}} F(w \circ \ell) G(w \circ \ell-\epsilon h \circ \ell) \\
& \quad \times \exp \left[\epsilon \int_{0}^{\ell_{T}} h(t) \mathrm{d} W_{t}(w)-\frac{1}{2} \epsilon^{2} \int_{0}^{\ell_{T}}\left|h^{\prime}(t)\right|^{2} \mathrm{~d} t\right] \mu(\mathrm{d} w) \lambda(\mathrm{d} \ell) .
\end{aligned}
$$

Differentiating this equality w.r.t. $\epsilon$ and setting $\epsilon=0$, we arrive at

$$
\begin{aligned}
& \iint_{\mathbb{W}_{M} \times \mathbb{S}_{T}} G(w \circ \ell) D_{h} F(w \circ \ell) \mu(\mathrm{d} w) \lambda(\mathrm{d} \ell) \\
& \quad=\iint_{\mathbb{W}_{M} \times \mathbb{S}_{T}} F(w \circ \ell)\left\{-D_{h} G(w \circ \ell)+G(w \circ \ell) \int_{0}^{\ell_{T}} h(t) \mathrm{d} W_{t}(w)\right\} \mu(\mathrm{d} w) \lambda(\mathrm{d} \ell),
\end{aligned}
$$

which gives the desired assertion.

Now we can investigate the gradient operator on $\Omega$.

Lemma 3.2. Let $F \in \mathscr{F} C_{b}^{\infty}$ and $\kappa \in \mathbb{R}$. Then for all $w \in \mathbb{W}_{M}$ and $\lambda$-almost all $\ell \in \mathbb{S}_{T}$, the map $h \mapsto D_{h} F(w \circ \ell)$ is a bounded linear functional on $\mathbb{H}_{M}^{(\kappa)}$.

Proof. Let $F \in \mathscr{F} C_{b}^{\infty}$ given by (3.1). By (3.2), the linearity is obvious. Since for $\lambda$-almost all $\ell \in \mathbb{S}_{T}$ we have $\ell_{t} \leq M$ for all $t \in[0, T]$, we obtain for all $w \in \mathbb{W}_{M}$ and $\lambda$-almost all $\ell \in \mathbb{S}_{T}$

$$
\begin{aligned}
& \left|D_{h} F(w \circ \ell)\right| \leq \sum_{i=1}^{n}\left\|\nabla_{i} f\right\|_{\infty}\left|h\left(\ell_{t_{i}}\right)\right| \\
& \quad \leq \sum_{i=1}^{n}\left\|\nabla_{i} f\right\|_{\infty} \int_{0}^{\ell_{t_{i}}}\left[\mathbb{P}\left(S_{T} \geq t\right)\right]^{-\kappa / 2}\left|h^{\prime}(t)\right|\left[\mathbb{P}\left(S_{T} \geq t\right)\right]^{\kappa / 2} \mathrm{~d} t \\
& \quad \leq \sum_{i=1}^{n}\left\|\nabla_{i} f\right\|_{\infty}\left(\int_{0}^{\ell_{t_{i}}}\left[\mathbb{P}\left(S_{T} \geq t\right)\right]^{-\kappa} \mathrm{d} t\right)^{1 / 2}\left(\int_{0}^{\ell_{t_{i}}}\left|h^{\prime}(t)\right|^{2}\left[\mathbb{P}\left(S_{T} \geq t\right)\right]^{\kappa} \mathrm{d} t\right)^{1 / 2} \\
& \quad \leq \sum_{i=1}^{n}\left\|\nabla_{i} f\right\|_{\infty}\left(\int_{0}^{\ell_{t_{i}}}\left[\mathbb{P}\left(S_{T} \geq t\right)\right]^{-\kappa} \mathrm{d} t\right)^{1 / 2}\|h\|_{\mathbb{H}_{M}^{(\kappa)}}
\end{aligned}
$$

To complete the proof, it remains to note that

$$
\int_{0}^{\ell_{t_{i}}}\left[\mathbb{P}\left(S_{T} \geq t\right)\right]^{-\kappa} \mathrm{d} t \leq\left[1 \vee\left[\mathbb{P}\left(S_{T} \geq \ell_{t_{i}}\right)\right]^{-\kappa}\right] \ell_{t_{i}} \leq\left[1 \vee\left[\mathbb{P}\left(S_{T} \geq \ell_{T}\right)\right]^{-\kappa}\right] \ell_{t_{i}}
$$

and

$$
\mathbb{P}\left(S_{T} \geq \ell_{T}\right)>0
$$


for $\lambda$-almost all $\ell \in \mathbb{S}_{T}$.

Let $F \in \mathscr{F} C_{b}^{\infty}$ and $\kappa \in \mathbb{R}$. Combining Lemma 3.2 with the Riesz representation theorem we find for all $w \in \mathbb{W}_{M}$ and $\lambda$-almost all $\ell \in \mathbb{S}_{T}$ that there exists a unique $D^{(\kappa)} F(w \circ \ell) \in \mathbb{H}_{M}^{(\kappa)}$ such that

$$
\left\langle D^{(\kappa)} F(w \circ \ell), h\right\rangle_{\mathbb{H}_{M}^{(\kappa)}}=D_{h} F(w \circ \ell), \quad F \in \mathscr{F} C_{b}^{\infty}, h \in \mathbb{H}_{M}^{(\kappa)} .
$$

For simplicity, we write $D F$ instead of $D^{(0)} F$. If $F \in \mathscr{F} C_{b}^{\infty}$ is given by (3.1), then it is easy to see that for all $w \in \mathbb{W}_{M}$ and $\lambda$-almost all $\ell \in \mathbb{S}_{T}$ we have

$$
\begin{gathered}
D^{(\kappa)} F(w \circ \ell)(t)=\sum_{i=1}^{n} \nabla_{i} f\left(w\left(\ell_{t_{1}}\right), \ldots, w\left(\ell_{t_{n}}\right)\right) \int_{0}^{t \wedge \ell_{t_{i}}}\left[\mathbb{P}\left(S_{T} \geq s\right)\right]^{-\kappa} \mathrm{d} s, \quad t \in[0, M], \\
\left\|D^{(\kappa)} F(w \circ \ell)\right\|_{\mathbb{H}_{M}^{(\kappa)}}^{2}=\sum_{i, j=1}^{n}\left\langle\nabla_{i} f\left(w\left(\ell_{t_{1}}\right), \ldots, w\left(\ell_{t_{n}}\right)\right), \nabla_{j} f\left(w\left(\ell_{t_{1}}\right), \ldots, w\left(\ell_{t_{n}}\right)\right)\right\rangle_{\mathbb{R}^{d}} \\
\times \int_{0}^{\ell_{t_{i}} \wedge \ell_{t_{j}}}\left[\mathbb{P}\left(S_{T} \geq s\right)\right]^{-\kappa} \mathrm{d} s .
\end{gathered}
$$

In particular, for $F \in \mathscr{F} C_{b}^{\infty}$ having the form (3.1), it holds that for all $w \in \mathbb{W}_{M}$ and $\lambda$-almost all $\ell \in \mathbb{S}_{T}$

$$
\begin{aligned}
& D F(w \circ \ell)(t)=\sum_{i=1}^{n}\left(t \wedge \ell_{t_{i}}\right) \nabla_{i} f\left(w\left(\ell_{t_{1}}\right), \ldots, w\left(\ell_{t_{n}}\right)\right), \quad t \in[0, M], \\
& \|D F(w \circ \ell)\|_{\mathbb{H}_{M}}^{2}=\sum_{i=1}^{n}\left(\ell_{t_{i}}-\ell_{t_{i-1}}\right)\left|\sum_{j=i}^{n} \nabla_{j} f\left(w\left(\ell_{t_{1}}\right), \ldots, w\left(\ell_{t_{n}}\right)\right)\right|^{2},
\end{aligned}
$$

where $t_{0}:=0$.

Recall that $\nu$ is the Lévy measure of the subordinator $\left(S_{t}\right)_{t \in[0, T]}$. We will use the following integrability condition:

$$
\int_{1}^{\infty} x^{p / 2} \nu(\mathrm{d} x)<\infty
$$

where $p>0$. In fact, since $\nu(1, \infty)<\infty,\left(\mathrm{H}_{p}\right)$ is automatically satisfied for $p \leq 0$.

Remark 3.3. It is well known that $\left(\overline{\mathrm{H}_{p}}\right)$ is equivalent to $\mathbb{E} S_{t}^{p / 2}<\infty$ for some (or all) $t \in[0, T]$, cf. [10, Theorem 25.3].

Let us introduce the following index of $S$ at the origin:

$$
\sigma_{0}:=\sup \left\{\alpha \geq 0: \lim _{u \downarrow 0} \frac{\phi(u)}{u^{\alpha}}=0\right\} \text {. }
$$

Noting that

$$
\lim _{u \downarrow 0} \frac{\phi(u)}{u}=\lim _{u \downarrow 0} \phi^{\prime}(u)=b+\lim _{u \downarrow 0} \int_{0}^{\infty} x \mathrm{e}^{-u x} \nu(\mathrm{d} x)=b+\int_{0}^{\infty} x \nu(\mathrm{d} x) \in(0, \infty],
$$

it follows that

$$
0 \leq \sigma_{0} \leq 1
$$

The following useful proposition is the subordinator counterpart of a result on general Feller processes from [11]. We defer its proof to the appendix (Section 4). 
Proposition 3.4. Let $\phi$ be given by (1.1). Then

$$
\sigma_{0}=\sup \left\{\alpha \geq 0: \limsup _{u \downarrow 0} \frac{\phi(u)}{u^{\alpha}}<\infty\right\}=\sup \left\{0 \leq \rho \leq 1: \int_{1}^{\infty} x^{\rho} \nu(\mathrm{d} x)<\infty\right\} \text {. }
$$

Remark 3.5. a) Clearly, $p / 2<\sigma_{0}$ implies $\left(\overline{\mathrm{H}_{p}}\right)$; conversely $\left(\overline{\mathrm{H}_{p}}\right)$ entails that either $p / 2 \leq \sigma_{0}$ or $p / 2>1$. In particular, $\left(\mathrm{H}_{2}\right)$ implies $\sigma_{0}=1$.

b) Since

$$
\phi^{\prime}(0+):=\lim _{u \downarrow 0} \phi^{\prime}(u)=b+\int_{(0,1]} x \nu(\mathrm{d} x)+\int_{1}^{\infty} x \nu(\mathrm{d} x),
$$

$\left(\mathrm{H}_{2}\right)$ is equivalent to $\phi^{\prime}(0+)<\infty$.

c) $\left(\mathrm{H}_{2}\right)$ is strictly stronger than $\sigma_{0}=1$. An example of a Bernstein function satisfying $\sigma_{0}=1$ but not $\left(\mathrm{H}_{2}\right)$ is (cf. [12, p. 316])

$$
\phi(u)=u \log \left(1+\frac{1}{u}\right), \quad u>0 .
$$

This function is even a complete Bernstein function. To see our claim, note that

$$
\sigma_{0}=1 \Longleftrightarrow \lim _{u \downarrow 0} \frac{\phi(u)}{u^{\alpha}}=0 \quad \text { for all } \alpha \in(0,1)
$$

and

$$
\left(\mathrm{H}_{2}\right) \text { fails } \Longleftrightarrow \phi^{\prime}(0+)=\infty \Longleftrightarrow \lim _{u \downarrow 0} \frac{\phi(u)}{u}=\infty \quad \text { (L'Hôspital's rule). }
$$

Lemma 3.6. Let $T<\infty$ and $M=\infty$. If $\sigma_{0}>0$ and $\theta>1 / \sigma_{0}$, then

$$
\int_{1}^{\infty}\left[\mathbb{P}\left(S_{T} \geq t\right)\right]^{\theta} \mathrm{d} t<\infty .
$$

Remark 3.7. Let $T<\infty$ and $S$ be an $\alpha$-stable subordinator $(0<\alpha<1)$. Obviously, $\sigma_{0}=\alpha$. It is well known that

$$
\mathbb{P}\left(S_{T} \geq t\right) \asymp t^{-\alpha}, \quad t \geq 1 .
$$

Therefore,

$$
\int_{1}^{\infty}\left[\mathbb{P}\left(S_{T} \geq t\right)\right]^{\theta} \mathrm{d} t<\infty \Longleftrightarrow \int_{1}^{\infty} t^{-\alpha \theta} \mathrm{d} t<\infty \Longleftrightarrow \theta>\frac{1}{\alpha}=\frac{1}{\sigma_{0}} .
$$

This means that Lemma 3.6 is sharp for $\alpha$-stable subordinators.

Proof of Lemma 3.6. Let $t \geq 1$; using

$$
\mathbb{1}_{[t, \infty)}\left(S_{T}\right) \leq \frac{2 S_{T}}{S_{T}+t} \text { and } \frac{x}{x+t}=t \int_{0}^{\infty}\left(1-\mathrm{e}^{-u x}\right) \mathrm{e}^{-t u} \mathrm{~d} u, \quad x \geq 0,
$$

together with Tonelli's theorem, we find

$$
\begin{aligned}
\mathbb{P}\left(S_{T} \geq t\right) & =\mathbb{E}\left[\mathbb{1}_{[t, \infty)}\left(S_{T}\right)\right] \\
& \leq 2 \mathbb{E}\left[\frac{S_{T}}{S_{T}+t}\right] \\
& =2 t \mathbb{E}\left[\int_{0}^{\infty}\left(1-\mathrm{e}^{-u S_{T}}\right) \mathrm{e}^{-t u} \mathrm{~d} u\right] \\
& =2 t \int_{0}^{\infty}\left(1-\mathrm{e}^{-T \phi(u)}\right) \mathrm{e}^{-t u} \mathrm{~d} u \\
& =2 \int_{0}^{\infty}\left(1-\mathrm{e}^{-T \phi(u / t)}\right) \mathrm{e}^{-u} \mathrm{~d} u
\end{aligned}
$$




$$
\leq 2 T \int_{0}^{\infty} \phi\left(\frac{u}{t}\right) \mathrm{e}^{-u} \mathrm{~d} u
$$

where the last estimate follows from the elementary inequality

$$
1-\mathrm{e}^{-x} \leq x, \quad x \in \mathbb{R}
$$

Pick $\alpha \in\left(1 / \sigma_{0}, \theta\right)$. By the first equality in (3.4), there exists a constant $c=c(\alpha)>0$ such that

$$
\phi(u) \leq c u^{1 / \alpha}, \quad 0<u \leq 1
$$

On the other hand, we have for all $u \geq 1$ that

$$
\begin{aligned}
\phi(u) & \leq b u+\left(\int_{0}^{1} x \nu(\mathrm{d} x)\right) u+\nu(x \geq 1) \\
& \leq\left[b+\int_{0}^{1} x \nu(\mathrm{d} x)+\nu(x \geq 1)\right] u=: C_{1} u .
\end{aligned}
$$

Thus, we get for $t \geq 1$-note that $1 / \alpha \in(0,1)$ -

$$
\begin{aligned}
\mathbb{P}\left(S_{T} \geq t\right) & \leq 2 T \int_{0}^{t} c\left(\frac{u}{t}\right)^{1 / \alpha} \mathrm{e}^{-u} \mathrm{~d} u+2 T \int_{t}^{\infty} C_{1} \frac{u}{t} \mathrm{e}^{-u} \mathrm{~d} u \\
& \leq 2 T\left(c \int_{0}^{t} u^{1 / \alpha} \mathrm{e}^{-u} \mathrm{~d} u+C_{1} \int_{t}^{\infty} u \mathrm{e}^{-u} \mathrm{~d} u\right) t^{-1 / \alpha} \\
& \leq 2 T\left(c \Gamma\left(\frac{1}{\alpha}+1\right)+C_{1}\right) t^{-1 / \alpha} \\
& =: C_{2} t^{-1 / \alpha}
\end{aligned}
$$

This, together with $\theta / \alpha>1$, implies that

$$
\int_{1}^{\infty}\left[\mathbb{P}\left(S_{T} \geq t\right)\right]^{\theta} \mathrm{d} t \leq C_{2}^{\theta} \int_{1}^{\infty} t^{-\theta / \alpha} \mathrm{d} t<\infty
$$

From the point view of functional analysis, the gradient operator is only useful if it is closable in some Banach space. To show this, the following two conditions will be used:

( $\mathrm{H}_{p}$ ) holds for some $p>0$;

$$
T<\infty, M=\infty, p \in(0,2], \sigma_{0}>0 \text { and } \kappa<1-1 / \sigma_{0} .
$$

Remark 3.8. a) Since $\sigma_{0} \leq 1$, we know that $\kappa<0$ is necessary for (A2).

b) Assume that $T<\infty$ and $M=\infty$. Then (A1) with $p \in(0,2]$ is strictly stronger than (A2). Indeed: First note that by Proposition 3.4 (A1) with $p \in(0,2]$ implies $\sigma_{0}=p / 2>0$, and so (A2) is fulfilled with $\kappa<1-2 / p$; moreover, for an $\alpha$-stable subordinator $(\alpha \in(0,1))$ (A2) holds with $\sigma_{0}=\alpha>0, \kappa<1-1 / \alpha$ and all $p \in(0,2]$, while (A1) holds if and only if $p<2 \alpha<2$.

Lemma 3.9. Let $F \in \mathscr{F} C_{b}^{\infty}$.

a) If (A1) holds, then $D_{h} F \in L^{p}\left(\mu^{S}\right)$ for any $h \in \mathbb{H}_{M}$ and $D F \in L^{p}\left(\Omega \rightarrow \mathbb{H}_{M} ; \mu^{S}\right)$.

b) If (A2) holds, then $D_{h} F \in L^{p}\left(\mu^{S}\right)$ for any $h \in \mathbb{H}_{M}^{(\kappa)}$ and $D^{(\kappa)} F \in L^{p}\left(\Omega \rightarrow \mathbb{H}_{M}^{(\kappa)} ; \mu^{S}\right)$.

Proof. By (3.3) and the elementary inequality

$$
\left(\sum_{i=1}^{n} a_{i}\right)^{p} \leq n^{(p-1) \vee 0} \sum_{i=1}^{n} a_{i}^{p}, \quad a_{i} \geq 0,
$$


we obtain for any $\kappa \in \mathbb{R}$ and $w \in \mathbb{W}_{M}$ and $\lambda$-almost all $\ell \in \mathbb{S}_{T}$

$$
\begin{aligned}
&\left|D_{h} F(w \circ \ell)\right|^{p} \leq n^{(p-1)^{+}}\|h\|_{\mathbb{H}_{M}^{(\kappa)}}^{p} \sum_{i=1}^{n}\left\|\nabla_{i} f\right\|_{\infty}^{p}\left(\int_{0}^{\ell_{t_{i}}}\left[\mathbb{P}\left(S_{T} \geq t\right)\right]^{-\kappa} \mathrm{d} t\right)^{p / 2}, \quad h \in \mathbb{H}_{M}^{(\kappa)}, \\
&\left\|D^{(\kappa)} F(w \circ \ell)\right\|_{\mathbb{H}_{M}^{(\kappa)}}^{p}=\sup _{\|h\|_{\mathrm{H}_{M}^{(\kappa)}} \leq 1}\left|D_{h} F(w \circ \ell)\right|^{p} \\
& \leq n^{(p-1) \vee 0} \sum_{i=1}^{n}\left\|\nabla_{i} f\right\|_{\infty}^{p}\left(\int_{0}^{\ell_{t_{i}}}\left[\mathbb{P}\left(S_{T} \geq t\right)\right]^{-\kappa} \mathrm{d} t\right)^{p / 2} .
\end{aligned}
$$

a) Assume that (A1) holds. Let $\kappa=0$. Then we have

$$
\int_{\mathbb{S}_{T}}\left(\int_{0}^{\ell_{t_{i}}}\left[\mathbb{P}\left(S_{T} \geq t\right)\right]^{-\kappa} \mathrm{d} t\right)^{p / 2} \lambda(\mathrm{d} \ell)=\int_{\mathbb{S}_{T}} \ell_{t_{i}}^{p / 2} \lambda(\mathrm{d} \ell)=\mathbb{E} S_{t_{i}}^{p / 2}<\infty,
$$

so that the first assertion follows.

b) Assume that (A2) holds. We use the Jensen inequality and Lemma 3.6 to get

$$
\begin{aligned}
\int_{\mathbb{S}_{T}}\left(\int_{0}^{\ell_{t_{i}}}\left[\mathbb{P}\left(S_{T} \geq t\right)\right]^{-\kappa} \mathrm{d} t\right)^{p / 2} \lambda(\mathrm{d} \ell) & \leq \int_{\mathbb{S}_{T}}\left(\int_{0}^{\ell_{T}}\left[\mathbb{P}\left(S_{T} \geq t\right)\right]^{-\kappa} \mathrm{d} t\right)^{p / 2} \lambda(\mathrm{d} \ell) \\
& \leq\left(\int_{\mathbb{S}_{T}}\left(\int_{0}^{\ell_{T}}\left[\mathbb{P}\left(S_{T} \geq t\right)\right]^{-\kappa} \mathrm{d} t\right) \lambda(\mathrm{d} \ell)\right)^{p / 2} \\
& =\left(\int_{0}^{\infty}\left[\mathbb{P}\left(S_{T} \geq t\right)\right]^{1-\kappa} \mathrm{d} t\right)^{p / 2} \\
& \leq\left(1+\int_{1}^{\infty}\left[\mathbb{P}\left(S_{T} \geq t\right)\right]^{1-\kappa} \mathrm{d} t\right)^{p / 2} \\
& <\infty
\end{aligned}
$$

For $p \geq 1$ we set

$$
L^{p+}\left(\mu^{S}\right):=\bigcup_{p<r \leq \infty} L^{r}\left(\mu^{S}\right) .
$$

As usual, we make the convention $1 / 0:=\infty$.

Theorem 3.10. Assume that (A1) holds with some $p \geq 1$ (resp. (A2) holds with some $p \in[1,2])$. Let $p /(p-1) \leq q \leq \infty$. For any $h \in \mathbb{H}_{M}$ (resp. $\left.h \in \mathbb{H}_{M}^{(\kappa)}\right)$, the directional derivative operator $D_{h}: \mathscr{F} C_{b}^{\infty} \rightarrow L^{p}\left(\mu^{S}\right)$ is closable in $L^{q}\left(\mu^{S}\right)$. Denote by $D_{h}$ its closure and let $D_{h}^{*}$ be its adjoint. Then

$$
\mathscr{D}\left(D_{h}\right) \cap L^{p+}\left(\mu^{S}\right) \subset \mathscr{D}\left(D_{h}^{*}\right)
$$

and for $G \in \mathscr{D}\left(D_{h}\right) \cap L^{p+}\left(\mu^{S}\right)$,

$$
D_{h}^{*} G=-D_{h} G+G \int_{0}^{S_{T}} h^{\prime}(t) \mathrm{d} W_{t} .
$$

Proof. a) Assume that (A1) holds for some $p \geq 1$.

a1) Let $\left\{F_{n}\right\}_{n \in \mathbb{N}} \subset \mathscr{F} C_{b}^{\infty}$ be a sequence such that $F_{n} \rightarrow 0$ in $L^{q}\left(\mu^{S}\right)$ and $D_{h} F_{n} \rightarrow Z$ in $L^{p}\left(\mu^{S}\right)$ as $n \rightarrow \infty$. In order to prove the closability of $D_{h}$, we have to show that $Z=0$. 
Fix $G \in \mathscr{F} C_{b}^{\infty}$. According to Lemma 3.9, $D_{h} G \in L^{p}\left(\mu^{S}\right)$. On the other hand, by Burkholder's inequality, we have for all $\theta \in(0, \infty)$

$$
\begin{aligned}
\mathbb{E}\left[\left|\int_{0}^{S_{T}} h^{\prime}(t) \mathrm{d} W_{t}\right|^{\theta}\right] & =\int_{\mathbb{S}_{T}} \mathbb{E}\left[\left|\int_{0}^{\ell_{T}} h^{\prime}(t) \mathrm{d} W_{t}\right|^{\theta}\right] \lambda(\mathrm{d} \ell) \\
& \leq \int_{\mathbb{S}_{T}} C_{\theta}\left(\int_{0}^{\ell_{T}}\left|h^{\prime}(t)\right|^{2} \mathrm{~d} t\right)^{\theta / 2} \lambda(\mathrm{d} \ell) \\
& \leq C_{\theta}\|h\|_{\mathbb{H}_{M}}^{\theta}
\end{aligned}
$$

where $C_{\theta}$ is a positive constant depending on $\theta$. Thus,

$$
D_{h}^{*} G=-D_{h} G+G \int_{0}^{S_{T}} h^{\prime}(t) \mathrm{d} W_{t} \in L^{p}\left(\mu^{S}\right) .
$$

The conjugate Hölder exponent $q^{\prime}$ of $q$ satisfies $1 \leq q^{\prime} \leq p$, and this implies that $D_{h}^{*} G \in$ $L^{q^{\prime}}\left(\mu^{S}\right)$. Therefore, we obtain

$$
\mathbb{E}[G Z]=\lim _{n \rightarrow \infty} \mathbb{E}\left[G D_{h} F_{n}\right]=\lim _{n \rightarrow \infty} \mathbb{E}\left[F_{n} D_{h}^{*} G\right]=0 .
$$

Since $G \in \mathscr{F} C_{b}^{\infty}$ is arbitrary and $\mathscr{F} C_{b}^{\infty}$ is dense in $L^{q}\left(\mu^{S}\right)$, we get $Z=0$.

a2) We proceed as in [7, Proof of Theorem 5.2]. Let $G \in \mathscr{D}\left(D_{h}\right) \cap L^{r}\left(\mu^{S}\right)$ for some $r \in(p, \infty]$. Since $D_{h}: \mathscr{F} C_{b}^{\infty} \rightarrow L^{p}\left(\mu^{S}\right)$ is closable in $L^{q}\left(\mu^{S}\right)$, there exists a sequence $\left\{G_{n}\right\}_{n \in \mathbb{N}} \subset \mathscr{F} C_{b}^{\infty}$ such that $G_{n} \rightarrow G$ in $L^{q}\left(\mu^{S}\right)$ and $D_{h} G_{n} \rightarrow D_{h} G$ in $L^{p}\left(\mu^{S}\right)$ as $n \rightarrow \infty$. With (3.6) it is easy to see that

$$
D_{h}^{*} G_{n}=-D_{h} G_{n}+G_{n} \int_{0}^{S_{T}} h^{\prime}(t) \mathrm{d} W_{t} \underset{n \rightarrow \infty}{\longrightarrow}-D_{h} G+G \int_{0}^{S_{T}} h^{\prime}(t) \mathrm{d} W_{t}
$$

in $L^{1}\left(\mu^{S}\right)$. Therefore, for any $F \in \mathscr{F} C_{b}^{\infty}$, by $D_{h} F \in L^{p}\left(\mu^{S}\right) \subset L^{q^{\prime}}\left(\mu^{S}\right)$, one has

$$
\begin{aligned}
\mathbb{E}\left[G D_{h} F\right] & =\lim _{n \rightarrow \infty} \mathbb{E}\left[G_{n} D_{h} F\right] \\
& =\lim _{n \rightarrow \infty} \mathbb{E}\left[F D_{h}^{*} G_{n}\right] \\
& =\mathbb{E}\left[F\left\{-D_{h} G+G \int_{0}^{S_{T}} h^{\prime}(t) \mathrm{d} W_{t}\right\}\right] .
\end{aligned}
$$

Since $G \in L^{r}\left(\mu^{S}\right)$ and (3.6) $)$, together with Hölder's inequality, imply that

$$
G \int_{0}^{S_{T}} h^{\prime}(t) \mathrm{d} W_{t} \in L^{p}\left(\mu^{S}\right)
$$

we get $G \in \mathscr{D}\left(D_{h}^{*}\right)$; in particular, (3.5) follows.

b) If (A2) holds for some $p \in[1,2]$, one can prove the claim as in the first case; we only need to replace (3.6) by

$$
\begin{aligned}
\mathbb{E}\left[\left|\int_{0}^{S_{T}} h^{\prime}(t) \mathrm{d} W_{t}\right|^{\theta}\right] & \leq \int_{\mathbb{S}_{T}} C_{\theta}\left(\int_{0}^{\ell_{T}}\left|h^{\prime}(t)\right|^{2} \mathrm{~d} t\right)^{\theta / 2} \lambda(\mathrm{d} \ell) \\
& \leq C_{\theta}\left(\int_{0}^{\infty}\left|h^{\prime}(t)\right|^{2}\left[\mathbb{P}\left(S_{T} \geq t\right)\right]^{\kappa} \mathrm{d} t\right)^{\theta / 2} \\
& =C_{\theta}\|h\|_{\mathbb{H}_{M}^{(\kappa)}}^{\theta},
\end{aligned}
$$

where we used, in the second inequality, $\kappa<0$. 
Theorem 3.11. Assume that (A1) holds for some $p \geq 1$ (resp. (A2) holds for some $p \in[1,2])$. Let $p /(p-1) \leq q \leq \infty$. The gradient operator $D: \mathscr{F} C_{b}^{\infty} \rightarrow L^{p}\left(\Omega \rightarrow \mathbb{H}_{M} ; \mu^{S}\right)$ (resp. $\left.D^{(\kappa)}: \mathscr{F} C_{b}^{\infty} \rightarrow L^{p}\left(\Omega \rightarrow \mathbb{H}_{M}^{(\kappa)} ; \mu^{S}\right)\right)$ is closable in $L^{q}\left(\mu^{S}\right)$. Denote its closure again by $D\left(\right.$ resp. $\left.D^{(\kappa)}\right)$. For any $h \in \mathbb{H}_{M}, \mathscr{D}(D) \subset \mathscr{D}\left(D_{h}\right)$; if $F \in \mathscr{D}(D)$ then $D_{h} F=$ $\langle D F, h\rangle_{\mathrm{H}_{M}}$ in $L^{p}\left(\mu^{S}\right)$ (resp. for any $\left.h \in \mathbb{H}_{M}^{(\kappa)}, \mathscr{D}\left(D^{(\kappa)}\right) \subset \mathscr{D}\left(D_{h}\right)\right)$; if $F \in \mathscr{D}\left(D^{(\kappa)}\right)$ then $D_{h} F=\left\langle D^{(\kappa)} F, h\right\rangle_{\mathbb{H}_{M}^{(\kappa)}}$ in $\left.L^{p}\left(\mu^{S}\right)\right)$.

Proof. We only prove the statement for the case that (A1) holds for some $p \geq 1$, since the proof for the other case is essentially similar.

a) Let $\left\{F_{n}\right\}_{n \in \mathbb{N}} \subset \mathscr{F} C_{b}^{\infty}$ be a sequence such that $F_{n} \rightarrow 0$ in $L^{q}\left(\mu^{S}\right)$ and $D F_{n} \rightarrow Y$ in $L^{p}\left(\Omega \rightarrow \mathbb{H}_{M} ; \mu^{S}\right)$ as $n \rightarrow \infty$. We will prove that $Y=0$. Fix an orthonormal basis $\left\{h_{i}\right\}_{i \in \mathbb{N}}$ of $\mathbb{H}_{M}$ and define

$$
\mathscr{C}\left(\mathrm{H}_{M}\right):=\left\{\sum_{i=1}^{m} G_{i} h_{i}: m \in \mathbb{N}, G_{i} \in \mathscr{F} C_{b}^{\infty}\right\} .
$$

It is easy to see that $\mathscr{C} \subset L^{q}\left(\Omega \rightarrow \mathbb{H}_{M} ; \mu^{S}\right)$ is a dense subset. Pick $G=\sum_{i=1}^{m} G_{i} h_{i} \in$ $\mathscr{C}\left(\mathbb{H}_{M}\right)$. By the orthogonal expansion of $D F_{n}$ in the orthonormal basis $\left\{h_{i}\right\}_{i \in \mathbb{N}}$ and the closability of $D_{h_{i}}: \mathscr{F} C_{b}^{\infty} \rightarrow L^{p}\left(\mu^{S}\right)$ in $L^{q}\left(\mu^{S}\right)$, cf. Theorem 3.10, we get

$$
\begin{aligned}
\mathbb{E}\langle G, Y\rangle_{\mathbb{H}_{M}} & =\lim _{n \rightarrow \infty} \mathbb{E}\left\langle G, D F_{n}\right\rangle_{\mathbb{H}_{M}} \\
& =\lim _{n \rightarrow \infty} \mathbb{E}\left[\sum_{i=1}^{m} \sum_{j=1}^{\infty}\left\langle h_{i}, h_{j}\right\rangle_{\mathbb{H}_{M}} G_{i} D_{h_{j}} F_{n}\right] \\
& =\lim _{n \rightarrow \infty} \sum_{i=1}^{m} \mathbb{E}\left[G_{i} D_{h_{i}} F_{n}\right] \\
& =\sum_{i=1}^{m} \lim _{n \rightarrow \infty} \mathbb{E}\left[G_{i} D_{h_{i}} F_{n}\right]=0 .
\end{aligned}
$$

Since $G \in \mathscr{C}$ is arbitrary and $\mathscr{C}$ is dense in $L^{q}\left(\Omega \rightarrow \mathbb{H}_{M} ; \mu^{S}\right)$, this implies that $Y=0$. Thus, $D$ is closable.

b) We will adopt the idea used in [7, Proof of Proposition 5.3]. Let $h \in \mathbb{H}_{M}$ and $F \in \mathscr{D}(D)$. Then there exists a sequence $\left\{F_{n}\right\}_{n \in \mathbb{N}} \subset \mathscr{F} C_{b}^{\infty}$ such that $F_{n} \rightarrow F$ in $L^{q}\left(\mu^{S}\right)$ and $D F_{n} \rightarrow D F$ in $L^{p}\left(\Omega \rightarrow \mathbb{H}_{M} ; \mu^{S}\right)$ as $n \rightarrow \infty$. Since

$$
\left|D_{h} F_{n}-D_{h} F_{m}\right|^{p}=\left|\left\langle D\left(F_{n}-F_{m}\right), h\right\rangle_{\mathbb{H}_{M}}\right|^{p} \leq\left\|D\left(F_{n}-F_{m}\right)\right\|_{\mathbb{H}_{M}}^{p}\|h\|_{\mathbb{H}_{M}}^{p}
$$

holds for all $m, n \in \mathbb{N}$, we conclude that $\left\{D_{h} F_{n}\right\}_{n \in \mathbb{N}}$ is a Cauchy sequence in $L^{p}\left(\mu^{S}\right)$. By the closedness of $D_{h}$, we have $F \in \mathscr{D}\left(D_{h}\right)$ and $D_{h} F_{n} \rightarrow D_{h} F$ in $L^{p}\left(\mu^{S}\right)$ as $n \rightarrow \infty$. To finish the proof, it remains to note that

$$
\begin{aligned}
\left\|D_{h} F-\langle D F, h\rangle_{\mathbb{H}_{M}}\right\|_{L^{p}\left(\mu^{S}\right)} & \leq\left\|D_{h} F-D_{h} F_{n}\right\|_{L^{p}\left(\mu^{S}\right)}+\left\|\left\langle D F_{n}, h\right\rangle-\langle D F, h\rangle_{\mathbb{H}_{M}}\right\|_{L^{p}\left(\mu^{S}\right)} \\
& \leq\left\|D_{h} F-D_{h} F_{n}\right\|_{L^{p}\left(\mu^{S}\right)}+\left\|D F_{n}-D F\right\|_{L^{p}\left(\Omega \rightarrow \mathbb{H}_{M} ; \mu^{S}\right)}\|h\|_{\mathbb{H}_{M}} \\
& \underset{n \rightarrow \infty}{\longrightarrow} 0 .
\end{aligned}
$$

If (A1) holds for $p=2$, then by Lemma 3.9 a), we can define a symmetric quadratic form on $\mathscr{F} C_{b}^{\infty}$ in the following way:

$$
\mathscr{E}(F, G):=\int_{\Omega}\langle D F, D G\rangle_{\mathbb{H}_{M}} \mathrm{~d} \mu^{S}<\infty, \quad F, G \in \mathscr{F} C_{b}^{\infty} .
$$


If $F \in \mathscr{F} C_{b}^{\infty}$ is of the form (3.1), then

$$
\mathscr{E}(F, F)=\mathbb{E}\left[\sum_{i=1}^{n}\left(S_{t_{i}}-S_{t_{i-1}}\right)\left|\sum_{j=i}^{n} \nabla_{j} f\left(W_{S_{t_{1}}}, \ldots, W_{S_{t_{n}}}\right)\right|^{2}\right],
$$

where $t_{0}:=0$.

Similarly, if (A2) holds for $p=2$, then with Lemma 3.9 b) we can define a symmetric quadratic form on $\mathscr{F} C_{b}^{\infty}$ :

$$
\mathscr{E}^{(\kappa)}(F, G):=\int_{\Omega}\left\langle D^{(\kappa)} F, D^{(\kappa)} G\right\rangle_{\mathbb{H}_{M}^{(\kappa)}} \mathrm{d} \mu^{S}<\infty, \quad F, G \in \mathscr{F} C_{b}^{\infty} .
$$

Moreover, for $F \in \mathscr{F} C_{b}^{\infty}$ given by (3.1), one has

$$
\begin{gathered}
\mathscr{E}^{(\kappa)}(F, F)=\mathbb{E}\left[\sum_{i, j=1}^{n}\left\langle\nabla_{i} f\left(W_{S_{t_{1}}}, \ldots, W_{S_{t_{n}}}\right), \nabla_{j} f\left(W_{S_{t_{1}}}, \ldots, W_{S_{t_{n}}}\right)\right\rangle_{\mathbb{R}^{d}}\right. \\
\left.\times \int_{0}^{S_{t_{i} \wedge t_{j}}}\left[\mathbb{P}\left(S_{T} \geq s\right)\right]^{-\kappa} \mathrm{d} s\right]
\end{gathered}
$$

As an immediate consequence of Theorem 3.11 with $p=q=2$, we obtain the following result concerning the Dirichlet form on $L^{2}\left(\mu^{S}\right)$ (see [4, 9] for more details on the theory of Dirichlet forms).

Proposition 3.12. If (A1) (resp. (A2) ) holds for $p=2$, then the form $\left(\mathscr{E}, \mathscr{F} C_{b}^{\infty}\right)$ (resp. $\left.\left(\mathscr{E}^{(\kappa)}, \mathscr{F} C_{b}^{\infty}\right)\right)$ is closable in $L^{2}\left(\mu^{S}\right)$, and the closure $(\mathscr{E}, \mathscr{D}(\mathscr{E}))($ resp. $(\mathscr{E}(\kappa), \mathscr{D}(\mathscr{E}(\kappa))))$ is a conservative symmetric Dirichlet form on $L^{2}\left(\mu^{S}\right)$.

We close this section by pointing out that it might be interesting (and also challenging) to consider various functional inequalities (cf. [14]) for the Dirichlet forms derived in Proposition 3.12 .

\section{Appendix}

In this section, we establish Proposition 3.4.

Proof of Proposition 3.4. a) Denote by $\sigma_{0}^{\prime}$ and $\sigma_{0}^{\prime \prime}$ the second and the third term in (3.4), respectively. Clearly, $\sigma_{0}^{\prime} \geq \sigma_{0}$. For any $\alpha<\sigma_{0}^{\prime}$, pick $\alpha^{\prime} \in\left(\alpha, \sigma_{0}^{\prime}\right)$. According to the definition of $\sigma_{0}^{\prime}$, one has

$$
\limsup _{u \downarrow 0} \frac{\phi(u)}{u^{\alpha^{\prime}}}<\infty
$$

and so

$$
\frac{\phi(u)}{u^{\alpha}}=\frac{\phi(u)}{u^{\alpha^{\prime}}} u^{\alpha^{\prime}-\alpha} \rightarrow 0 \quad \text { as } u \downarrow 0 .
$$

This means that $\alpha \leq \sigma_{0}$. Letting $\alpha \uparrow \sigma_{0}^{\prime}$, we get $\sigma_{0}^{\prime} \leq \sigma_{0}$. Therefore, $\sigma_{0}^{\prime}=\sigma_{0}$.

b) We prove that $\sigma_{0} \leq \sigma_{0}^{\prime \prime}$. Without loss of generality, we may assume that $0<\sigma_{0} \leq 1$. Fix any $\alpha \in\left(0, \sigma_{0}\right) \subset(0,1)$ and pick $\alpha^{\prime} \in\left(\alpha, \sigma_{0}\right)$. By the definition of $\sigma_{0}$,

$$
\lim _{u \downarrow 0} \frac{\phi(u)}{u^{\alpha^{\prime}}}=0
$$

and so there exists a constant $c=c\left(\alpha^{\prime}\right)>0$ such that

$$
\phi(u) \leq c u^{\alpha^{\prime}}, \quad 0<u<1 .
$$


Recall the identity

$$
x^{\alpha}=\frac{\alpha}{\Gamma(1-\alpha)} \int_{0}^{\infty}\left(1-\mathrm{e}^{-x u}\right) u^{-\alpha-1} \mathrm{~d} u, \quad x>0 .
$$

Combining this with Tonelli's theorem, the following inequality

$$
\int_{1}^{\infty}\left(1-\mathrm{e}^{-x u}\right) \nu(\mathrm{d} x) \leq \nu(1, \infty) \wedge\left(\int_{0}^{\infty}\left(1-\mathrm{e}^{-x u}\right) \nu(\mathrm{d} x)\right) \leq \nu(1, \infty) \wedge \phi(u), \quad u>0
$$

and (4.1), we obtain that

$$
\begin{aligned}
\int_{1}^{\infty} x^{\alpha} \nu(\mathrm{d} x) & =\frac{\alpha}{\Gamma(1-\alpha)} \int_{0}^{\infty}\left(\int_{1}^{\infty}\left(1-\mathrm{e}^{-x u}\right) \nu(\mathrm{d} x)\right) u^{-\alpha-1} \mathrm{~d} u \\
& \leq \frac{\alpha}{\Gamma(1-\alpha)} \int_{0}^{\infty}[\nu(1, \infty) \wedge \phi(u)] u^{-\alpha-1} \mathrm{~d} u \\
& \leq \frac{\alpha}{\Gamma(1-\alpha)} \int_{0}^{1} \phi(u) u^{-\alpha-1} \mathrm{~d} u+\frac{\alpha \nu(1, \infty)}{\Gamma(1-\alpha)} \int_{1}^{\infty} u^{-\alpha-1} \mathrm{~d} u \\
& \leq \frac{\alpha c}{\Gamma(1-\alpha)} \int_{0}^{1} u^{\alpha^{\prime}-\alpha-1} \mathrm{~d} u+\frac{\alpha \nu(1, \infty)}{\Gamma(1-\alpha)} \int_{1}^{\infty} u^{-\alpha-1} \mathrm{~d} u<\infty
\end{aligned}
$$

This implies that $\alpha \leq \sigma_{0}^{\prime \prime}$. Since $\alpha<\sigma_{0}$ is arbitrary, we conclude that $\sigma_{0} \leq \sigma_{0}^{\prime \prime}$.

c) It remains to show that $\sigma_{0}^{\prime \prime} \leq \sigma_{0}$. As in part b), we can assume that $\sigma_{0}^{\prime \prime} \in(0,1]$. Fix any $\rho \in\left(0, \sigma_{0}^{\prime \prime}\right)$ and pick $\rho^{\prime} \in\left(\rho, \sigma_{0}^{\prime \prime}\right) \subset(0,1)$. Then

$$
\int_{1}^{\infty} x^{\rho^{\prime}} \nu(\mathrm{d} x)<\infty
$$

Assume that $u \in(0,1)$. Since

$$
1-\mathrm{e}^{-x u}<\left\{\begin{array}{ll}
x u, & \text { if } 0<x \leq 1, \\
x u \leq(x u)^{\rho^{\prime}}, & \text { if } 1<x \leq u^{-1}, \\
1<(x u)^{\rho^{\prime}}, & \text { if } x>u^{-1}
\end{array} \Longrightarrow 1-\mathrm{e}^{-x u}< \begin{cases}x u, & \text { if } 0<x \leq 1, \\
(x u)^{\rho^{\prime}}, & \text { if } x>1,\end{cases}\right.
$$

it follows that

$$
\begin{aligned}
\phi(u) & \leq b u+\int_{(0,1]} x u \nu(\mathrm{d} x)+\int_{1}^{\infty}(x u)^{\rho^{\prime}} \nu(\mathrm{d} x) \\
& \leq\left[b+\int_{(0,1]} x \nu(\mathrm{d} x)+\int_{1}^{\infty} x^{\rho^{\prime}} \nu(\mathrm{d} x)\right] u^{\rho^{\prime}}=: C\left(\rho^{\prime}\right) u^{\rho^{\prime}} .
\end{aligned}
$$

From this we get

$$
\limsup _{u \downarrow 0} \frac{\phi(u)}{u^{\rho}} \leq \limsup _{u \downarrow 0} C\left(\rho^{\prime}\right) u^{\rho^{\prime}-\rho}=0 .
$$

Consequently, we obtain $\rho \leq \sigma_{0}$ and then $\sigma_{0}^{\prime \prime} \leq \sigma_{0}$ by letting $\rho \uparrow \sigma_{0}^{\prime \prime}$.

\section{REFERENCES}

[1] R.H. Cameron, W.T. Martin: Transformations of Wiener integrals under translations. Ann. Math. 45 (1944) 386-396.

[2] C.-S. Deng, R.L. Schilling: On shift Harnack inequalities for subordinate semigroups and moment estimates for Lévy processes. Preprint, arXiv: 1412.6700.

[3] B.K. Driver: A Cameron-Martin type quasi-invariance theorem for Brownian motion on a compact Riemannian manifold. J. Funct. Anal. 110 (1992) 272-376.

[4] M. Fukushima, Y. Oshima, M. Takeda: Dirichlet Forms and Symmetric Markov Processes (2nd ed). De Gruyter, Studies in Mathematics 19, Berlin 2011. 
[5] I. Gentil, P. Maheux: Super-Poincaré and Nash-type inequalities for subordinated semigroups. To appear in Semigroup Forum, DOI: 10.1007/s00233-014-9648-2.

[6] M. Gordina, M. Röckner, F.-Y. Wang: Dimension-independent Harnack inequalities for subordinated semigroups. Potential Anal. 34 (2011) 293-307.

[7] E.P. Hsu: Quasi-invariance of the Wiener measure on the path space over a compact Riemannian manifold. J. Funct. Anal. 134 (1995) 417-450.

[8] E.P. Hsu: Stochastic Analysis on Manifolds. Am. Math. Soc., Graduate Studies in Mathematics 38, Providence (RI) 2001.

[9] M. Röckner, Z.-M. Ma: Introduction to the Theory of (Non-Symmetric) Dirichlet Forms. Springer, Universitext, New York 1992.

[10] K. Sato: Lévy Processes and Infinitely Divisible Distributions (2nd ed). Cambridge University Press, Cambridge 2013.

[11] R.L. Schilling: Growth and Hölder conditions for the sample paths of Feller processes. Probab. Theory Rel. Fields 112 (1998) 565-611.

[12] R.L. Schilling, R. Song, Z. Vondraček: Bernstein Functions. Theory and Applications (2nd ed). De Gruyter, Studies in Mathematics 37, Berlin 2012.

[13] R.L. Schilling, J. Wang: Functional inequalities and subordination: stability of Nash and Poincaré inequalities. Math. Z. 272 (2012) 921-936.

[14] F.-Y. Wang: Functional Inequalities, Markov Semigroups and Spectral Theory. Science Press, Beijing, New York 2005.

[15] F.-Y. Wang: Coupling for Ornstein-Uhlenbeck processes with jumps. Bernoulli 17 (2011) 11361158.

[16] F.-Y. Wang: Integration by parts formula and shift Harnack inequality for stochastic equations. Ann. Probab. 42 (2014) 994-1019.

[17] F.-Y. Wang, C. Yuan: Poincaré inequality on the path space of Poisson point processes. J. Theor. Probab. 23 (2010) 824-833.

CHINA

(C.-S. Deng) School of Mathematics and Statistics, Wuhan University, Wuhan 430072,

Current address: TU Dresden, Fachrichtung Mathematik, Institut für Mathematische Stochastik, 01062 Dresden, Germany

E-mail address: dengcs@whu.edu.cn

(R.L. Schilling) TU Dresden, Fachrichtung Mathematik, Institut für Mathematische Stochastik, 01062 Dresden, Germany

E-mail address: rene.schilling@tu-dresden.de 